\title{
Balance control, flexibility, and cardiorespiratory fitness among older Tai Chi practitioners
}

Youlian Hong, Jing Xian Li, P D Robinson

\begin{abstract}
Background-Tai Chi Chuan (TTC) exercise has beneficial effects on the components of physical condition and can produce a substantial reduction in the risk of multiple falls. Previous studies have shown that short term TCC exercise did not improve the scores in the single leg stance test with eyes closed and the sit and reach test. There has apparently been no research into the effects of TCC on total body rotation flexibility and heart rate responses at rest and after a three minute step test.
\end{abstract}

Methods-In this cross sectional study, 28 male TCC practitioners with an average age of 67.5 years old and 13.2 years of TCC exercise experience were recruited to form the TCC group. Another 30 sedentary men aged 66.2 were selected to serve as the control group. Measurements included resting heart rate, left and right single leg stance with eyes closed, modified sit and reach test, total body rotation test (left and right), and a three minute step test.

Results-Compared with the sedentary group, the TCC group had significantly better scores in resting heart rate, three minute step test heart rate, modified sit and reach, total body rotation test on both right and left side $(p<0.01)$, and both right and left leg standing with eyes closed $(p<0.05)$. According to the American Fitness Standards, the TCC group attained the 90th percentile rank for sit and reach and total body rotation test, right and left.

Conclusion-Long term regular TCC exercise has favourable effects on the promotion of balance control, flexibility, and cardiovascular fitness in older adults.

(Br F Sports Med 2000;34:29-34)

Keywords: Tai Chi; balance; falls; flexibility; cardiovascular fitness; aged

Falls, a real problem in public health, are the main cause of accidental death in the elderly. ${ }^{1}$ Poor balance capacity with age results in increased risk of falls and fracture for people over $65 .^{2}{ }^{3}$ Exercise and other forms of physical activity are known to provide a myriad of health benefits to older adults. ${ }^{4}$ Studies have found that older people respond favourably to exercise, and perform better than an age matched group of older non-exercisers in fitness components, such as balance, flexibility, reaction time, and strength. ${ }^{56}$ Diminished functioning in these aspects have been shown to be major contributors to falls in older persons. ${ }^{7}$

Tai Chi Chuan (TCC) was developed originally as a martial arts form and has been used for centuries in China as an exercise for health in a wide age range but particularly in the elderly. The basic exercise of TCC is a series of individual movements linked together in a continuous manner that flow smoothly from one movement to another. Deep breathing and mental concentration are also required to achieve harmony between body and mind. Thus, TCC is not only a physical activity, but also one that involves the training of mental control. ${ }^{8}$ People use TCC for development of mind-body interaction, breathing regulation with body movement, hand-eye coordination, and calmness. ${ }^{9}$ In addition, TCC can be practised at any time and in any place because it needs neither much space nor any equipment. The simple, soft, and fluid movements are ideal for older people regardless of previous exercise experience. Today, millions of people in China are practising TCC; they perform it in public parks, on streets, or wherever it can be. Moreover, it has spread to many countries world wide and is extensively accepted as an exercise form for health and fitness.

A number of studies have been performed to examine the effects of TCC exercise on the physical condition of elderly people. In a cross sectional study, Tse and Bailey ${ }^{10}$ compared older adult TCC practitioners with older adult non-practitioners on five balance tests. Their study showed that the TCC practitioners scored higher than TCC non-practitioners in the test of single leg stance with eyes open, but not in the test of single leg stance with eyes closed. Lai et $a l^{11}$ evaluated the effects of two years of regular TCC exercise on the maintenance of cardiovascular function in older subjects and found a smaller decline in the maximal oxygen uptake over the two years for TCC practitioners than for their sedentary counterparts.

Lan et $a l^{12}$ performed a cross sectional study to assess the potential effects of TCC on cardiovascular function, flexibility, and body composition in the elderly. The long term TCC practitioners showed higher peak oxygen uptake, better scores in the stand and reach test and a lower percentage of body fat than their sedentary counterparts. Schaller ${ }^{13}$ determined the effects of 10 weeks of the westernised form of TCC on balance control, flexibility, and blood pressure in older persons. The TCC intervention resulted in a significant improvement in the scores of single leg stance with eyes open, but not in the single leg stance with eyes 
closed. Moreover, the scores of the sit and reach test for TCC practitioners were not improved. It is possible that the duration of exercise was not sufficient to improve the balance capacity with eyes closed or the flexibility. Wolf $e t a l^{14}$ compared the biomedical, functional, and psychological indicators of frailty between a 15 week TCC or a computerised balance training intervention in older persons. The TCC intervention resulted in a lowered blood pressure before and after a 12 minute walk. A reduced fear of falling responses and intrusiveness responses, or perceived ability to do all that one would like to do, was also observed. Wolfson et $a l^{15}$ studied the effect of six months of low intensity TCC on the maintenance of gains in balance and strength in older adults. Recently, Jacobson et $a l^{16}$ reported significantly better balance control (tilting board test), strength of knee extension (maximal voluntary extension test), and kinaesthetic sense (glenohumeral media rotation at $90^{\circ}$ ) after 12 weeks of TCC compared with the sedentary control group.

It appears from the literature that TCC does not improve performance in tests of single leg stance with eyes closed. However, the studies that yielded this conclusion were based on a cross sectional comparison of TCC practitioners with a large discrepancy in TCC experience (from 1 to 20 years), ${ }^{10}$ or were based on an application of a self modified and westernised TCC form of short term duration. ${ }^{9}$ Moreover, the literature shows conflicting findings on the effects of TCC on trunk and hamstring flexibility in elderly people. The cross sectional study $^{12}$ that employed TCC practitioners with an average of more than 10 years of TCC experience showed significantly better stand and reach scores for the TCC practitioners than for the sedentary control subjects, whereas Schaller ${ }^{13}$ reported no beneficial effect of TCC on sit and reach scores after a short term intervention. No studies have examined the potential effects of TCC on total body rotation flexibility, which is related to hip, trunk, and shoulder joint, ${ }^{17}$ and to heart rate responses before and after exercise. In classical TCC, the mind is tranquil but alert; body movements are slow, smooth, and well coordinated with a lowered centre of gravity as the various forms of the exercise are undertaken. Owing to the nature of its performance, TCC has generally been assumed to have significantly favourable effects on flexibility, balance control, and cardiorespiratory fitness in older people after long term exercise. However, studies on the effects of long term classical TCC on physical fitness in the elderly are lacking in the literature. Our aim in this study was to observe whether older adults who practised TCC over a long time ( 10 years or more) performed better than their counterparts in single leg stance with eyes closed, sit and reach, total body rotation, and heart rate both at rest and after a three minute step test. The scores both on the sit and reach test and total body rotation (left and right) test were used to determine flexibility. The scores on heart rate at rest and after the three minute step test were used to determine cardiorespiratory fitness. Lan et $a l^{12}$ used the stand and reach test, whereas, this study employed sit and reach as an indicator of trunk and hamstring flexibility because it is best correlated with hamstring flexibility ${ }^{18}$. The seated version was used and is recommended by the American College of Sports Medicine ${ }^{18}$ for trunk and hamstring flexibility. Thus the findings of this study should contribute to our knowledge of aging and the effects of physical activity, such as TTC. As it is difficult to perform a follow up investigation for a long term study of more than 10 years, a cross sectional approach was adopted. The hypotheses stated in this study were as follows. Long term TCC practitioners perform better than their sedentary counterparts in tests of $(a)$ balance in terms of single leg stance with eyes closed, (b) trunk and hamstring flexibility in terms of sit and reach, (c) total body flexibility in terms of total body rotation test (left and right), and (d) cardiorespiratory fitness in terms of heart rate at rest and after a three minute step test.

Approval for this study was given by the local clinical research ethics committee.

\section{Methods}

SUBJECTS

Recruitment criteria were as follows: (a) age 65 or over; (b) no history of significant cardiovascular, pulmonary, metabolic, musculoskeletal, and other chronic diseases; (c) living in the community with a normally active life style; $(d)$ TCC experience of about 10 years or more and no involvement in any regular sports and physical activity other than TCC in the previous five years for the TCC group, and no exercise training programme for at least five years for the control group; (e) to eliminate the influence of gender on the physical fitness scores, only male subjects were selected to form the TCC and sedentary groups.

The TCC practitioners were recruited from one of the largest classical Yang Style Tai Chi Clubs. Hundreds of people practice TCC in this club every day. The experienced TCC practitioners perform TCC individually or in groups, while the beginners practice TCC in groups instructed by qualified teachers.

The sedentary control group was recruited from a large housing estate in the city. With the assistance of the estate management office, letters that described the purpose of the study, the physical requirements for the participants, measurement items, and measurement procedure were distributed to each household to invite the senior family members, if any, to participate in this study. The same letters were also distributed to the senior TCC practitioners of the TCC club.

A total of 114 persons, 54 TCC practitioners and 60 sedentary non-practitioners, expressed their willingness to participate in this project. Questionnaires were then distributed to these people in order to collect information on personal particulars, history of illness, and sports and physical activity involvement. For TCC practitioners, information about their TCC experience (years), average exercise frequency (times a week), and the duration of 
Table 1 General description of subjects

\begin{tabular}{lcccc}
\hline & $\begin{array}{c}\text { TCC group } \\
(n=28)\end{array}$ & $\begin{array}{c}\text { Sedentary group } \\
(n=30)\end{array}$ & $t$ & \multicolumn{1}{c}{$t$} \\
\hline Age (years) & $67.5(5.8)$ & $66.2(6.5)$ & 0.802 & 0.426 \\
Body weight $(\mathrm{kg})$ & $65.7(9.9)$ & $68.6(5.5)$ & 1.391 & 0.170 \\
Body height $(\mathrm{cm})$ & $166.4(7.9)$ & $168.5(5.1)$ & 1.211 & 0.231 \\
Body mass index $\left(\mathrm{kg} / \mathrm{m}^{2}\right)$ & $22.7(3.6)$ & $24.2(2.9)$ & 1.753 & 0.085 \\
Exercise experience (years) & $13.2(3.7)$ & 0 & - & - \\
Frequency (times a week) & $5.1(1.1)$ & 0 & - & - \\
Duration (min per session) & $55.6(10.3)$ & 0 & - & - \\
\hline
\end{tabular}

Values are mean (SD). TCC, Tai Chi Chuan.

each exercise session (minutes per session) were also obtained. From the assessment of the collected questionnaires, there were only 28 TCC practitioners, and 30 sedentary nonpractitioners who met the requirements. These formed the TCC practitioner group and sedentary control group respectively. Subjects were informed about the study, and their consent to participate was obtained. Table 1 gives the general description of the TCC practitioners and sedentary subjects. No significant difference was found in age, body weight, body height, and body mass index between the two groups.

MEASUREMENTS

Measurements were mainly the physical fitness components of balance, flexibility, and heart rate by the seven test items described below. The content of the tests was explained to the subjects and they were given time to prepare with a warm up so that their best performance could be achieved. Tests were arranged in the morning before any other exercise. Subjects were asked to wear loose clothes and comfortable shoes with low heels. Body height and weight were also measured. Each subject went through the tests in the same sequence, test 1 to test 7 . After test 3 had been completed, a 10 minute period was provided for each subject to warm up in preparation for the next test, which was the modified sit and reach test.

The protocols of the seven tests were as follows.

Test 1: resting heart rate was recorded for each subject before any test began.

Test 2: single right leg standing with eyes closed.

Test 3: single left leg standing with eyes closed.

Balance control was assessed by single limb stance timed tests with the eyes closed. These forms of testing have been extensively used in assessment of balance and exercise with the elderly, and internal consistency reliability of 0.85 to 0.95 has been reported for this method. ${ }^{19}$ During the test, subjects were asked to stand on a firm surface for 60 seconds, and look straight ahead. A stopwatch was used to record in seconds the duration of standing. A maximum of three trials was allowed for each test for subjects to reach the goal of 60 seconds. The score of their best performance on each test was recorded. If the subjects reached the goal of 60 seconds, no further trials were conducted for that particular test. ${ }^{10}$

Test 4: modified sit and reach. The flexibility of trunk and hamstring was measured by a sit and reach test protocol modified as described by Hoeger and Hoeger ${ }^{17}$ using the Acuflex I Tester. A test-retest reliability of 0.83 and interclass reliability of 0.98 and 0.99 have been reported when it has been used with the elderly. ${ }^{20}{ }^{21}$

Test 5: total body rotation, left. The flexibility of total body rotation to the left was measured by the protocol provided by Hoeger and Hoeger ${ }^{17}$ using the Acuflex II.

Test 6: total body rotation, right. The flexibility of total body rotation to the right was measured in the same manner as that for the left.

Test 7: step test. Cardiovascular ability was measured by a three minute step test in accordance with the YMCA protocol. ${ }^{22}$ The test was conducted on a 12 inch high bench with a stepping rate of 24 steps/min for three minutes. After the exercise had been completed, the subjects were immediately seated and heart rate was counted for one minute, starting within five seconds of the end of the exercise.

All subjects completed test 1 to test 7 successfully.

ANALYSIS

$t$ tests for independent sampling using a two tailed test were used to determine if the performance of the TCC group was different from that of the sedentary group. Statistical significance was set at $\mathrm{p}<0.05$.

\section{Results}

Figure 1 shows the results of tests on right and left leg stance with eyes closed. The TCC group achieved significantly higher scores in right and left single leg stance with eyes closed $(\mathrm{p}<0.05)$ than the sedentary control group (9.64 (7.53) $v 6.28$ (4.17) seconds for right and 9.41 (6.80) $v 5.96$ (3.96) seconds for left leg). These results could not be detected in short term TCC practitioners in previously reported studies.

The data obtained from the sit and reach test showed that long term regular TCC practitioners had better $(\mathrm{p}<0.01)$ trunk and hamstring flexibility than their sedentary counterparts $(39.18(4.77) v 30.80(4.98) \mathrm{cm})$. This beneficial effect of TCC on sit and reach scores was not observed after a short term intervention. ${ }^{13}$ In addition, significantly $(\mathrm{p}<0.01)$ better scores

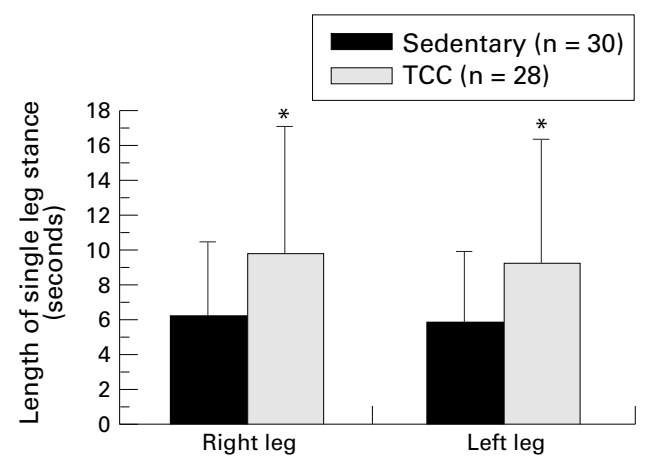

Figure 1 Comparison of measures in single leg standing with eyes closed between Tai Chi Chuan (TCC) and sedentary groups. ${ }^{\star} p<0.05$ compared with the sedentary group. 


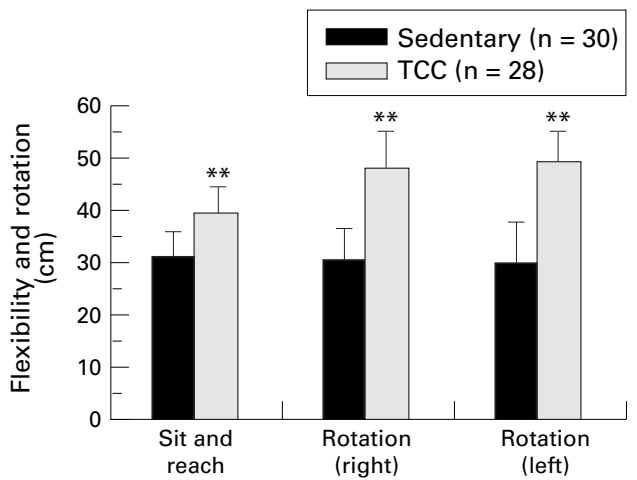

Figure 2 Comparison of body flexibility measures between the Tai Chi Chuan (TCC) and sedentary groups. ${ }_{\star \star *} p<0.01$ compared with the sedentary group.

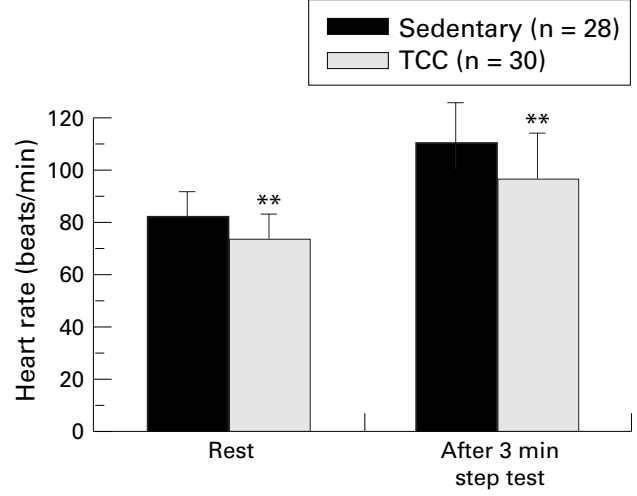

Figure 3 Comparison of heart rate measures between the Tai Chi Chuan (TCC) and sedentary groups. ${ }^{\star \star} p<0.01$ compared with the sedentary group.

for TCC practitioners than for the sedentary subjects were also seen in the total body rotation test $(47.73(6.55) v 30.59(5.61) \mathrm{cm}$ for right and $48.32(6.42) v 29.73(7.71) \mathrm{cm}$ for left rotation). Figure 2 illustrates the results from the sit and reach test and total body rotation test.

Significantly better scores for heart rate both at rest (69.01 (9.62) v 76.88 (9.32) beats $/ \mathrm{min})$ $(\mathrm{p}<0.01)$ (fig 3$)$ and after a three minute step test $(90.64(16.63) v 103.15$ (14.78) beats/ $\min ; \mathrm{p}<0.01)$ were found in the TCC group when compared with the sedentary group. The time of recovery of heart rate in TCC subjects was faster than in their sedentary counterparts, suggesting that the TCC exercise improved cardiovascular function in the elderly.

\section{Discussion}

BALANCE CONTROL

Balance is one of the components required for execution of postural control. Balance capacity decreases with age, which results in increased risk of falls and fractures in elderly people. ${ }^{23}$ Previous studies found beneficial effects of TCC on balance in older adults through comparing experienced TCC practitioners with non-practitioners in cross sectional ${ }^{10}$ or longitudinal ${ }^{13}$ studies based on the tests of single leg (right and left) standing with eyes open. The present study found that the scores of the TCC group in single leg (right and left) standing with eyes closed were also higher than that of the sedentary group, disagreeing with the results of Tse and Bailey ${ }^{10}$ who found no significant difference between TCC practitioners and sedentary subjects on single leg standing with eyes closed, for both the right and left leg. They suggested that maintenance of balance with vision occluded is not a normal life experience and is therefore equally difficult for people regardless of their activity level. In fact, classical TCC needs to be performed continuously and slowly, with knee flexion and weight shifting, straight and extended head and trunk, as well as unilateral weight bearing with constant shifting. All these are associated with the use of internal feedback to control the centre of mass, thus benefiting balance ability regardless of whether eyes are open or closed. In the study of Tse and Bailey, ${ }^{10}$ the TCC subjects included those who had only one year of TCC experience. This fact may indicate that long term TCC produces more benefit for balance control and thus for postural control of older persons. This warrants further study.

\section{FLEXIBILITY}

The development and maintenance of some level of flexibility are important components of a general health enhancement programme, and are even more so during the aging process. This study shows that long term regular TCC practitioners possessed better trunk and hamstring flexibility than their sedentary counterparts. This is confirmation of the finding by Lan et $a l,{ }^{12}$ who reported significantly superior performance by older TCC practitioners with more than 10 years of TCC experience than their sedentary counterparts in hip joint flexibility, monitored by the stand and reach test scores. Schaller ${ }^{13}$ reported that a 10 week TCC intervention did not improve trunk and hamstring flexibility, and Levandoski LJ (unpublished thesis) also found that a 10 week TCC intervention did not improve trunk or lower extremity flexibility. The possible explanation is that the exercise used by Schaller to train older people was a movement form modified from classical TCC so that it could easily be learned in weeks instead of years. This form may not include those elements present in classical TCC that involve hip flexion and knee extension. As a result of increased trunk and hamstring flexibility, sit and reach scores are also improved. Another explanation is that the development and maintenance of flexibility in the elderly may require more time and frequency of exercise.

The total body rotation test was developed specifically to measure the flexibility related to hip, trunk, and shoulder joints, and serves as an indicator of everyday movements in turning. ${ }^{17}$ Classical TCC contains the movements of combined rotation of head, trunk, and extremities, and asymmetrical diagonal arm and leg movements about the waist. All these elements can benefit hip, trunk, and arm rotation ability, and therefore the total body rotation test scores. According to the American Fitness Standards ${ }^{17}$ the percentile rank for sit and reach, total body rotation test (left), and total body rotation (right) obtained by the TCC group were 90, 90, and 80 respectively. These 
scores were much higher than those obtained by the sedentary control group $(60,40$, and 40 ), showing the significant benefits of classical TCC that contributed to the overall flexibility fitness of these older people.

HEART RATE

Studies investigating the effects of TCC on cardiovascular function in the elderly are limited. Lai et $a l^{11}$ studied two year trends in cardiovascular function among elderly TCC practitioners and sedentary subjects. In that study, cardiovascular function, including maximal oxygen uptake and heart rate during the test, were measured at the initial session and after two years of TCC practice. The results showed that regular TCC may delay the decline of cardiovascular function, as indicated by the smaller decrease in maximal oxygen uptake in TCC practitioners compared with sedentary subjects. Another study on the effects of TCC on cardiovascular function in the elderly was reported by Schaller, ${ }^{13}$ who found that simplified TCC exercise for 10 weeks in the elderly could significantly improve systolic and diastolic blood pressure. These two studies and the findings from the present study show the beneficial effects of TCC exercise on cardiovascular function in older adults.

The cross sectional approach was adopted in this study to assess the potential effect of TCC on physical fitness in the elderly. A longitudinal approach with before and after testing is recognised as an ideal method for measuring long term training effects of physical activity and exercise in older people. Its advantage over the cross sectional approach is that it enables one to control confounding hidden factors, such as heredity, and minimise the changes caused by aging. However, drop out of subjects is a common confounding factor in longitudinal studies, especially when the objective is to determine such a long term effect with aging as 10 years of training. The comparison in this study was based on a cross sectional approach, and has shown significant differences, providing indirect evidence that long term TCC exercise has the potential to play an important role in improving and maintaining physical fitness in the elderly.

Bortz $^{23}$ stated that cardiovascular endurance, muscle mass, muscle strength, and functional ability are interrelated and have all been reported to decline with advancing age and disuse. However, this decline in fitness and functional ability can be reduced or prevented by physical activity. Aniansson et $a l^{4}$ suggested that exercise programmes offer older persons a means of keeping physically active, even when they have moderate disorders, and that improved physical fitness may help prevent age dependent impairments. Previous and current studies, both cross sectional ${ }^{10}$ and longitudinal, ${ }^{11}{ }^{13}{ }^{14}$ have shown the beneficial functions of TCC.

SUMMARY

TCC has been defined as an aerobic exercise of moderate intensity. ${ }^{7}$ The degree of movement, although less strenuous than swimming, is more subtle and precise. This gives the TCC practitioner greater satisfaction and a sense of achievement. These satisfactory feelings come from two aspects. One relates to the ease of study and another is the emotional component. In this study, the long term TCC practitioners performed better in tests of balance, flexibility, and cardiovascular function than their sedentary counterparts. Some of these superior results have not been observed in short term TCC practitioners. Although the progressive decline with age in balance, flexibility, and functional capacity of the cardiovascular system is a natural state, the findings of this study show that practising TCC long term and regularly may decrease the rate of decline. Long term and regular practice of TCC should be recommended for older adults.

This study was supported by a direct grant for research from the Chinese University of Hong Kong.

Contributors: Y H contributed to the development of the study design, coordinated the collection and collation of the data, assisted in the interpretation of the data, and wrote the initial draft assisted in the interpretation of the data, and wrote the initial draft
of the paper. J X L contributed to the development of the study of the paper. J X L contributed to the development of the study
design and collation of the data, assisted in the interpretation of design and collation of the data, assisted in the interpretation of
the data, and participated in the writing of the paper. P D R conthe data, and participated in the writing of the paper. P D R con-
tributed to the coordination of data collection, assisted in the tributed to the coordination of data collection, assisted in the
interpretation of the data, participated in writing and discussing the paper, and approved the final manuscript.

1 Overstall PW, Johnson AL, Exton-Smith AN. Instability and falls in the elderly. Age Ageing 1978;7(suppl):92-6.

2 Nicken $\mathrm{H}$. Intrinsic factors in falling among the elderly. Arch Intern Med 1985;145:1089-93.

3 Perry B. Falls among the elderly: a review of the methods and conclusion of epidemiologic studies. F Am Geriatr Soc 1982;30:367-71.

4 Pescatello LS, DiPietro L. Physical activity in older adults. Sports Med 1993;15:353-64.

5 Lord SR, Castell S. Physical activity program for older persons: effect on balance, strength, neuromuscular control, and reaction time. Arch Phys Med Rehabil 1994;75: $648-52$.

6 Skelton DA, Young A, Greig CA, et al. Effects of resistance training on strength, power and selected functional abilities of women aged 75 and older. I Am Geriatr Soc 1995;43:1081-7.

7 Lord SR, Russell DC, Iran WW. Physiological factors associated with falls in an elderly population. 7 Am Geriatr Soc 1991;39:1194-200.

8 Kutner NG, Barnhart H, Wolf SL, et al. Self-report benefits of Tai Chi practice by older adults. F Gerontol 1997;52:2426 .

9 Wolf SL, Kutner NG, Green RC, et al. The Atlanta FICSIT study: two exercise interventions to reduce frailty in elders. 7 Am Geriatr Soc 1993;41:329-32.

10 Tse SK, Bailey DM. Tai Chi and postural control in the well elderly. Am f Occup Ther 1992;46:295-300.

11 Lai JS, Lan C, Wong MK, et al. Two-year trends in cardiorespiratory function among older Tai Chi Chuan practitioners and sedentary subjects. F Am Geriatr Soc 1995;43:1222-7.

2 Lan C, Lai JS, Wong MK, et al. Cardiorespiratory function, flexibility and body composition among geriatric Tai Chi Chuan Practitioners. Arch Phys Med Rehabil 1996;77:61216

13 Schaller KJ. Tai Chi Chih: an exercise option for older adults. Fournal of Gerontology Nursing 1996;22:12-17.

14 Wolf SL, Barnhart HX, Kutner NG, et al. Reducing frailty and falls in older persons: an investigation of Tai Chi and computerised balance training. F Am Geriatr Soc 1996;44: 489-97.

15 Wolfson L, Whipple R, Derby C, et al. Balance and strength training in older adults: intervention gains and Tai Chi maintenance. $\mathcal{F}$ Am Geriatr Soc 1996;44:498-506.

16 Jacobson BH, Chen HC, Cashel C, et al. The effect of Tai Jacobson BH, Chen HC, Cashel C, et al. The effect of Tai
Chi Chuan training on balance, kinesthetic sense, and strength. Percept Mot Skills 1997;84:27-33.

17 Hoeger WWK, Hoeger SA. Lifetime physical fitness and wellness: a personalised program. 3rd ed. Englewood, CO: Morton Publishing Company, 1992.

18 Pate RR, Shephard RJ. Characteristics of physical fitness in youth. In: Lamb DR, Murray R, Gisolfi CV, et al, eds. Perspectives in exercise science and sports medicine: volume 2 , youth, exercise, and sports. Indianapolis, Ind: Benchmark Press, 1989:1-45.

19 Rikli RE, Edwards DJ. Effects of a three-year exercise program on motor function and cognitive processing speed in older women. Res $Q$ Exerc Sports 1991;62:61-7.

20 Frekany GA, Leslie DK. Effects of an exercise program on selected flexibility measurements of senior citizens. Gerontologist 1975;15:182-3. 
21 Shephard RJ, Berridge M, Montelpare W. On the generality of the "sit and reach" test: an analysis of flexibility data for an aging population. Res Q Exerc Sports 1990;61:32630.

22 American College of Sports Medicine. ACSM's guidelines for exercise testing and prescription. 5th ed. Baltimore: Williams
\& Wilkins, 1995.

3 Bortz WM. Disuse and aging. FAMA 1982;248:1203-8. 24 Aniansson A, Ljungberg P, Rundgren P, et al. Effect of a training programme for pensioners on condition and muscular strength. Archives of Gerontology and Geriatrics $1984 ; 3: 229-41$

\section{Take home message}

Assessment of balance, flexibility, and cardiorespiratory fitness in older long term Tai Chi practitioners and sedentary counterparts indicates that the former improve and maintain good physical condition, particularly with respect to balance capacity, which has not been observed after short term Tai Chi exercise in older adults. This suggests that regular and long term practice of Tai Chi has a positive effect on balance, flexibility, and cardiorespiratory fitness in the elderly.

\section{Commentary}

There has been a lot of recent interest in methods of reducing the risk factors associated with falls, actual falls, and fall induced injuries in both scientific and health promotion literature.

A number of national guidelines ${ }^{12}$ have suggested the development of multifactorial interventions that include exercise involving muscle strengthening and balance work, especially Tai Chi. ${ }^{3}$ This cross sectional study shows that prolonged Tai Chi practice (over ten years) can have a significant enhancing effect on balance, flexibility, and cardiovascular function in older age. It also adds supporting evidence to an increasingly popular form of exercise that is suitable for older people. However, its applicability to an already frail older person, with poor balance, starting Tai Chi has not been established, and care must be taken that the exercise is sufficiently adapted to be safe for vulnerable older people. ${ }^{4}$

D SKELTON

Queen Elizabeth the Queen Mother Research Fellow, Imperial College of Science and Technology and Medicine,

Research into Ageing

1 Feder G, Cryer C, Donovan S. Guidelines for the prevention of falls in older people. Department of General Practice and Primary Care, Queen Mary and Westfield College, London and the South East Institute of Public Health, 1998.

2 Simey P, Pennington B. Physical activity and management of fractures and falling. A framework for practice. London: Health Education Authority, 1999.

3 Province MA, Hadley EC, Hornbrook MC, et al. The effects of exercise on falls in elderly patients. A pre-planned meta-analysis of the FICSIT Trials. $\mathcal{F A M A}$ 1955;173:1341-7.

4 Skelton DA, Dinan S. Exercise for fall management: rationale for an exercise programme aimed at reducing postural instability. Physiotherapy: Theory and Practice 1999;15:in press. 